\title{
Effect of Surcharge on the Stability of Rock Slope under Complex Conditions
}

\author{
Jiewen Tu*, Aiping Tang, Yuejun Liu and Ketong Liu \\ School of Civil Engineering, Harbin Institute of Technology, Harbin, 150090- China
}

Received 15 May 2013; Accepted 25 July 2013

\begin{abstract}
In this paper, a general analytical expression for the factor of safety of the rock slope against plane failure is proposed, incorporating most of the practically occurring under complex conditions such as depth of tension crack, depth of water in tension crack, seismic loads and surcharge. Several special cases of this expression are established, which can be found similarly to those reported in the literature. A detailed parametric analysis is presented to study the effect of surcharge on the stability of the rock slope for practical ranges of main parameters such as depth of tension crack, depth of water in tension crack, the horizontal seismic coefficient and the vertical seismic coefficient. The parametric analysis has shown that the factor of safety of the rock slope decreases with increase in surcharge for the range of those parameters in this paper. It is also shown that the horizontal seismic coefficient is the most important factor which effects on the factor of safety in the above four influence factors. The general analytical expression proposed in this paper and the results of the parametric analysis can be used to carry out a quantitative assessment of the stability of the rock slopes by engineers and researchers.
\end{abstract}

Keywords: Rock Slope; Tension Crack; Surcharge; Seismic loads; Factor of Safety

\section{Introduction}

The rock slope can be failure and instability by earthquake, which is one of the common earthquake disasters [1, and 2]. In recent years, it has caused rock slope failure in Wenchuan earthquake [3, 4, and 5] and Yushu earthquake [6] in China. The rock slope failure is characterized by extensive distribution, large quantity and great hazards. It will not only cause huge casualties and direct economic losses, but also cause traffic disruption, which can affect the relief and postearthquake recovery and other works. At present, the static stability analysis method of slope has been mature, which usually include limit equilibrium method, numerical analysis method and probability method. However, the dynamic stability analysis of the slope is still in the immature stage. And the main research methods are pseudo-static method, Newmark sliding block analysis method, dynamic finite element time history analysis method and so on [1].The quasi-static method has been widely applied for its convenience, and it is extremely popular with engineers and researchers [7]. Hence, this paper uses the quasi-static method to analysis the effect of surcharge on the stability of rock slope under complex conditions.

The rock slope can be failure due to its geotechnical properties, geological structure conditions, other internal factors and the various external conditions such as depth of tension crack, depth of water in tension crack, seismic loads, surcharge, etc $[8,9$, and 10]. And the rock slope failures in

* E-mail address: tujiewen@163.com

ISSN: 1791-2377 @ 2013 Kavala Institute of Technology. All rights reserved. one or the combination of some idealized types, such as circular failure, plane failure, wedge failure and toppling failure [11]. A plane failure usually occurs in hard or soft rock slopes with well defined discontinuities and jointing [12]. The evaluation of stability of the natural rock slopes becomes very essential for the safe design, especially when the slopes are situated close to residential areas or when structures are built on these slopes. Therefore, this paper attempts to propose a general analytical expression considering most of the field parameters under complex conditions such as surcharge, water pressure and seismic loads. The general analytical expression the analysis results of the effect of surcharge on the stability of rock slope under complex conditions can be used to carry out a quantitative assessment of the stability of the rock slopes by engineers and researchers.

\section{Analytical formulation}

The geometric factors of a typical rock slope are shown as Fig.1. And it shows a rock slope of height $H$ inclined to the horizontal at an angle $\beta$. The sliding rock $A_{1} A_{2} A_{3} A_{4}$ is separated by a vertical tension crack $A_{2} A_{3}$ of depth $\mathrm{z}$ and the failure plane $A_{1} A_{2}$, which is inclined to the horizontal at an angle $\alpha$. The tension crack is filled with water to a depth $Z_{\mathrm{w}}$. The weight of the sliding rock mass block is and $B\left(=A_{3} A_{4}\right)$ is the top width of the slope [12]. The slope is subjected to surcharge $q$. The horizontal and vertical seismic loads $\left(k_{\mathrm{h}} W\right.$ and $k_{\mathrm{v}} W, \quad k_{\mathrm{h}} q B$ and $\left.k_{\mathrm{v}} q B\right)$ are considered to act on the slope, where $k_{\mathrm{h}}$ and $k_{\mathrm{v}}$ are horizontal and vertical seismic coefficients, respectively. The horizontal force due 
to water pressure in the tension crack is $U_{1}$, and the uplift force due to water pressure on the failure plane is $U_{2}$. The slope stability is studied as a two-dimensional problem, considering a slice of unit thickness through the slope, as suggested by Hoek [12]. It is also important to know that this analysis considers only force equilibrium without considering any resistance to sliding at the lateral boundaries of the sliding block [12].

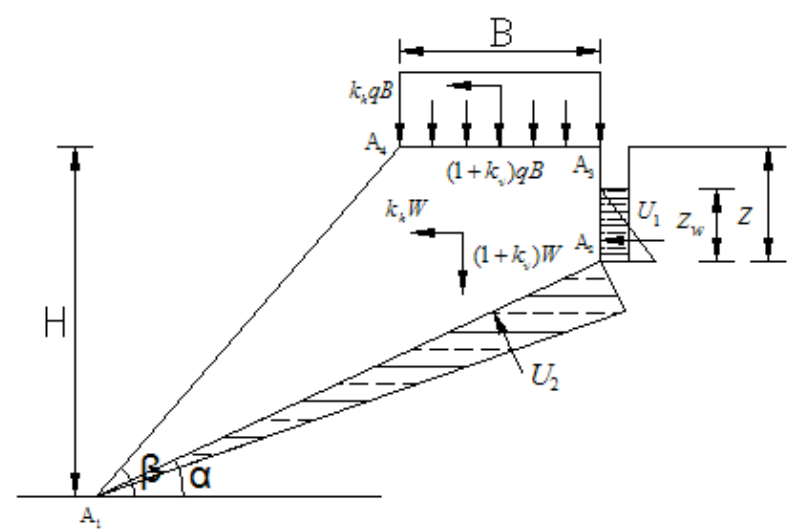

Fig. 1. Geometric factors of a typical rock slope as

The factor of safety $F_{\mathrm{s}}$ of the rock slope can be defined

$F_{\mathrm{s}}=\frac{F_{r}}{F_{i}}$

where $F_{\mathrm{r}}$ is the total force available to resist sliding, and $F_{i}$ is the total force tending to induce sliding.

$F_{\mathrm{r}}=S A$

where $\mathrm{s}$ is the shear strength of the sliding failure plane, and $A$ is the area of the base $A_{1} A_{2}$ of the sliding rock block given as

$A=H\left(1-\frac{z}{H}\right) \frac{1}{\sin \alpha}$

The top width $B$ is calculated as

$B=H\left\{\left(1-\frac{z}{H}\right) \cot \alpha-\cot \beta\right\}$

The shear strength of the sliding failure plane can be defined in terms of the Mohr-Coulomb failure criterion as:

$s=c+\sigma_{n} \tan \phi$

where $\sigma_{n}$ is the normal stress on the failure plane, and $c$ and $\phi$ are cohesion and angle of internal friction of the joint material. From Eqs.(2) and (5) can become as

$F_{r}=c A+F_{\mathrm{n}} \tan \phi$
Where $F_{n}=\sigma_{n} A$ is the normal force on the failure plane. Considering equilibrium of forces acting on the rock block, $F_{n}$ is obtained as

$$
F_{\mathrm{n}}=(W+q B)\left\{\left(1+k_{\mathrm{v}}\right) \cos \alpha-k_{\mathrm{h}} \sin \alpha\right\}-U_{1} \sin \alpha-U_{2}
$$

The weight of the sliding rock block is

$$
W=\frac{1}{2} \gamma H^{2}\left[\left\{1-\left(\frac{z}{H}\right)\right\} \cot \alpha-\cot \beta\right]
$$

The horizontal force due to water pressure in the tension crack is

$U_{1}=\frac{1}{2} \gamma_{w} z_{w} \times z_{w}=\frac{1}{2} \gamma_{w} z_{w}^{2}$

where $\gamma_{w}$ is the unit weight of water.

The uplift force due to water pressure on the failure plane is

$U_{2}=\frac{1}{2} \gamma_{w} z_{w} H\left(1-\frac{z}{H}\right) \frac{1}{\sin \alpha}$

Substituting values from Eqs.(3), (4) and (7) through (10) into Eq.(6)

$$
\begin{aligned}
& F_{r}=c H\left(1-\frac{z}{H}\right) \frac{1}{\sin \alpha}+\left[( 1 + k _ { \mathrm { v } } ) \left[\frac { 1 } { 2 } \gamma H ^ { 2 } \left[\left\{1-\left(\frac{z}{H}\right)^{2}\right\} .\right.\right.\right. \\
& \left.\cot \alpha-\cot \beta]+q H\left\{\left(1-\frac{z}{H}\right) \cot \alpha-\cot \beta\right\}\right] \frac{\cos (\theta+\alpha)}{\cos \theta} \\
& \left.-\frac{1}{2} \gamma_{w} z_{w}^{2} \sin \alpha-\frac{1}{2} \gamma_{w} z_{w} H\left(1-\frac{z}{H}\right) \frac{1}{\sin \alpha}\right] \tan \phi
\end{aligned}
$$

where

$$
\theta=\tan ^{-1}\left(\frac{k_{h}}{1+k_{v}}\right)
$$

From Fig.1, the total force tending to induce sliding is calculated as

$F_{i}=\left(1+k_{v}\right)\left[\frac{1}{2} \gamma H^{2}\left[\left\{1-\left(\frac{z}{H}\right)^{2}\right\} \cot \alpha-\cot \beta\right]+q H\right.$

$\left.\cdot\left\{\left(1-\frac{z}{H}\right) \cot \alpha-\cot \beta\right\}\right] \cdot \frac{\sin (\theta+\alpha)}{\cos \theta}+\frac{1}{2} \gamma_{w} z_{w}^{2} \cos \alpha$

Substituting $F_{\mathrm{r}}$ and $F_{i}$ from Eqs.(11) and (13), respectively, into Eq.(1)

$F S=\left[2 c^{*} P+\left[\left(1+k_{v}\right)\left(Q+2 q^{*} R\right) \frac{\cos (\theta+\alpha)}{\cos \theta}-\frac{z_{w}^{* 2}}{\gamma^{*}} \sin \alpha\right.\right.$

$\left.\left.-\frac{z_{w}^{*}}{\gamma^{*}} P\right] \times \tan \phi\right] /\left[\left(1+k_{v}\right)\left(Q+2 q^{*} R\right) \frac{\sin (\theta+\alpha)}{\cos \theta}+\frac{z_{w}^{* 2}}{\gamma^{*}} \cos \alpha\right]$ 
where $c^{*}=c / \gamma H, z^{*}=z / H, z_{w}^{*}=z_{w} / H$, $\gamma^{*}=\gamma / \gamma_{w}$ and $q^{*}=q / \gamma H$ are non-dimensional forms of $c, z, z_{w}, \gamma$ and $q$, respectively, and

$$
\begin{aligned}
& P=\left(1-z^{*}\right) \frac{1}{\sin \alpha} \\
& Q=\left(1-z^{* 2}\right) \cot \alpha-\cot \beta \\
& R=\left(1-z^{*}\right) \cot \alpha-\cot \beta
\end{aligned}
$$

Eq. (14) is the general expression for $F_{\mathrm{s}}$ of the rock slop against plane failure. It can be used to get other expressions of some special cases and observe the effect of any individual parameter on the safety of the rock slope and to carry out a detailed parametric study as required in a specific field situation.

\section{Cases study}

The general equation [Eq. (14)] developed for $F_{\mathrm{s}}$ of the rock slop against plane failure can have some special cases as explained below.

Case 1. The joint material is cohesionless whether subjected to surcharge or not, and there is no seismic forces and water in the tension crack, that is, $c^{*}=0, \phi \neq 0$, $q^{*}=0$ or $q^{*} \neq 0, k_{h}=0, k_{v}=0, \theta=0, z_{w}{ }^{*}=0$. Here, Eq. (14) can be both reduced to the expression given as Eq. (18).

$$
F S=\frac{\tan \phi}{\tan \alpha}
$$

Case 2. The joint material is cohesive, and there is no seismic forces and water in the tension crack, that is, $c^{*} \neq 0, \phi=0, q^{*} \neq 0, k_{h}=0, k_{v}=0, \theta=0, z_{w}^{*}=0$. Here, Eq. (14) becomes

$$
F_{s}=\frac{2 c^{*} P}{\left(Q+2 q^{*} R\right) \sin \alpha}
$$

Case 3. The joint material is $c-\phi$ material, and there is no seismic forces and water in the tension crack, that is, $c^{*} \neq 0, \phi \neq 0, q^{*} \neq 0, k_{h}=0, k_{v}=0, \theta=0, z_{w}^{*}=0$. Here, Eq. (14) becomes

$$
F_{s}=\frac{2 c^{*} P+\left[\left(Q+2 q^{*} R\right) \cos \alpha\right] \times \tan \phi}{\left(Q+2 q^{*} R\right) \sin \alpha}
$$

Case 4. The joint material is $c-\phi$ material, and there is no seismic forces, that is, $c^{*} \neq 0$, $\phi \neq 0, q^{*} \neq 0, k_{h}=0, k_{v}=0, \theta=0, z_{w}^{*} \neq 0$. Here, Eq. (14) becomes
$F_{s}=\frac{2 c^{*} P+\left[\left(Q+2 q^{*} R\right) \cos \alpha-\frac{z_{w}^{* 2}}{\gamma^{*}} \sin \alpha-\frac{z_{w}^{*}}{\gamma^{*}} P\right] \times \tan \phi}{\left(Q+2 q^{*} R\right) \sin \alpha+\frac{z_{w}^{* 2}}{\gamma^{*}} \cos \alpha}$

Case 5. The joint material is $c-\phi$ material, and there is only horizontal seismic force, that is, $c^{*} \neq 0$, $\phi \neq 0, q^{*} \neq 0, k_{h} \neq 0, k_{v}=0, \theta=\tan ^{-1}\left(k_{h}\right), z_{w}^{*} \neq 0$. Here, Eq. (14) becomes

$$
\begin{aligned}
& F_{s}=\left\{2 c^{*} P+\left[\left(Q+2 q^{*} R\right) \frac{\cos (\theta+\alpha)}{\cos \theta}-\frac{z_{w}^{* 2}}{\gamma^{*}} \sin \alpha\right.\right. \\
& \left.\left.-\frac{z_{w}^{*}}{\gamma^{*}} P\right] \times \tan \phi\right\} /\left[\left(Q+2 q^{*} R\right) \frac{\sin (\theta+\alpha)}{\cos \theta}+\frac{z_{w}^{* 2}}{\gamma^{*}} \cos \alpha\right]
\end{aligned}
$$

For a generalized case when the joint material is $c-\phi$ material, that is, $c^{*} \neq 0, \phi \neq 0, q^{*} \neq 0, k_{h} \neq 0, k_{v} \neq 0$, $\theta=\tan ^{-1}\left[k_{h} /\left(1+k_{v}\right)\right], z_{w}^{*} \neq 0$. Eq. (14) is applicable. It should be noted that some of the above special cases have been presented in similar forms in the literature $[12,13$, and $14]$.

\section{Parametric Analysis}

A parametric study is carried out to analysis the effect of surcharge $\left(q^{*}\right)$ on the stability of the rock slope in terms of the factor of safety. There are many factors affect the stability of rock slope, and this paper only focus on the depth of tension crack $\left(z^{*}\right)$, the depth of water in tension crack $\left(z_{w}^{*}\right)$, the horizontal seismic coefficient $\left(k_{h}\right)$ and the vertical seismic coefficient $\left(k_{v}\right)$. And the basic parameters are $\alpha=30^{\circ}, \beta=50^{\circ}, c^{*} \neq 0.1, \phi=25^{\circ}$ and $\gamma^{*}=2.5$, however, the ranges of the parameters are $q^{*}=0 \sim 2.0, z^{*}=0 \sim 0.3$, $z_{w}^{*}=0 \sim 0.2, k_{h}=0 \sim 0.2$ and $k_{v}=0 \sim 0.2$.

\subsection{The Influence Parameter of $z^{*}$}

Fig.2 shows the variation of $F_{s}$ with $q^{*}$ for different nondimensional values of $z^{*}$, which $z^{*}=0,0.15$ and 0.30 , considering specific values of governing parameters in their nondimensional form as: $\alpha=30^{\circ}, \quad \beta=50^{\circ}$, $z_{w}^{*}=0, \gamma^{*}=2.5, \quad c^{*}=0.1, \phi=25^{\circ}, k_{h}=0.1 \quad$ and $k_{v}=0.05$. It is observed that the values of $F_{s}$ decreases with the increase of $q^{*}$. From the results in Fig.2, it is also observed that $F_{s}$ is greater than unity for $z^{*}=0,0.15$ and 0.30 at lower values of $q^{*}$, but the decrease rate of $F_{s}$ is relatively higher for lower values of $q^{*}$. For example, for $z^{*}=0.15$, as $q^{*}$ increases from 0 to $0.5, F_{s}$ decreases by 0.28 , whereas for increase in $q^{*}$ from 0.5 to $1, F_{s}$ decreases by 0.11 . It can be noted that for lower values of 
$q^{*}, F_{s}$ is higher for smaller value of $z^{*}$, whereas for higher $q^{*}$ values, $F_{s}$ becomes higher for greater value of $z^{*}$.

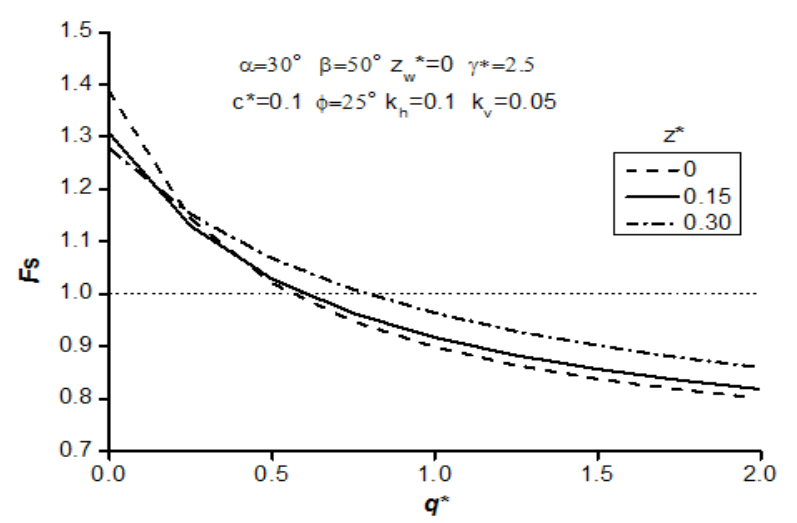

Fig. 2. Variation of $F_{s}$ with $q^{*}$ for different values of $z^{*}$

\subsection{The Influence Parameter of $z_{w}^{*}$}

Fig.3 shows the variation of $F_{s}$ with $q^{*}$ for different nondimensional values of $z_{w}^{*}$, which $z_{w}^{*}=0,0.1$, and 0.2 , considering specific values of governing parameters in their nondimensional form as: $\alpha=30^{\circ}, \beta=50^{\circ}, z^{*}=0.2$, $\gamma^{*}=2.5, c^{*}=0.1, \phi=25^{\circ}, k_{h}=0.1$ and $k_{v}=0.05$. From the Fig.3, it can be seen that the values of $F_{s}$ decreases with the increase of $q^{*}$ for all three cases and its rate of decrease is relatively higher for lower values of $q^{*}$. For example, for $z_{w}^{*}=0.1, F_{s}$ decreases by 0.23 for an increase in $q^{*}$ from 0 to 0.5 , whereas decrease in $F_{s}$ is 0.1 as $q^{*}$ increases from 0.5 to 1 . It is also observed that for any $q^{*}, F_{s}$ decreases with increase in the value of $z_{w}^{*}$. Hence, a perfectly stable rock slope becomes unsafe by increasing $q^{*}$, and the deterioration in $F_{s}$ is rather rapid for all three cases. As seen before, $F_{s}$ depends significantly on the parameter of $z_{w}^{*}$, and engineers and researchers should pay attention to drainage in practical engineering.

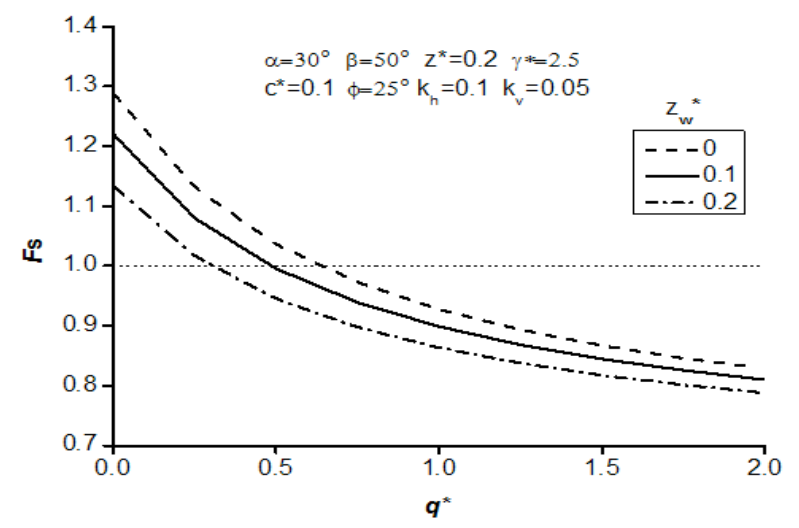

Fig. 3. Variation of $F_{s}$ with $q^{*}$ for different values of $z_{w}{ }^{*}$
4.3 The Influence Parameter of $k_{h}$

Fig.4 shows the variation of $F_{s}$ with $q^{*}$ for different values of horizontal seismic force, $k_{h}=0,0.1$, and 0.2 , considering specific values of governing parameters in their nondimensional form as: $\alpha=30^{\circ}, \beta=50^{\circ}, z^{*}=0.2$, $\gamma^{*}=2.5, c^{*}=0.1, \phi=25^{\circ}, z_{w}^{*}=0.1$ and $k_{v}=0$. From the Fig.4, it can be observed that $F_{s}$ depends significantly on the parameter of $k_{h}$. The value of $F_{s}$ is not less than 1.0 when the value of $k_{h}$ is 0 , however, the value of $F_{s}$ is nearly less than 1.0 when the value of $k_{h}$ is 0.2 . It can be seen that the values of $F_{s}$ decreases with the increase of $q^{*}$ for all three cases and $F_{s}$ is greater than unity for any value of $k_{h}$ at lower values of $q^{*}$, but it decreases being higher for lower values of $q^{*}$. For example, for $k_{h}=0.1, \quad F_{s}$ decreases by 0.24 for an increase in $q^{*}$ from 0 to 0.5 , whereas decrease in $F_{s}$ is 0.1 as $q^{*}$ increases from 0.5 to 1 . As seen before, engineers and researchers should pay attention to the horizontal seismic load in practical engineering.

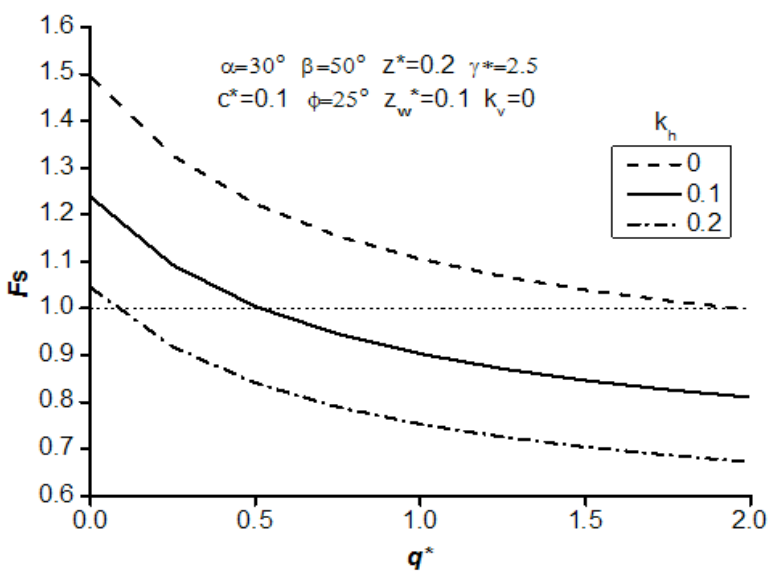

Fig. 4. Variation of $F_{s}$ with $q^{*}$ for different values of $k_{h}$

\subsection{The Influence Parameter of $k_{v}$}

Fig. 5 shows the variation of $F_{s}$ with $q^{*}$ for different values of vertical seismic force, $k_{v}=0,0.1$, and 0.2 , considering specific values of governing parameters in their nondimensional form as: $\alpha=30^{\circ}, \beta=50^{\circ}, z^{*}=0.2$, $\gamma^{*}=2.5, c^{*}=0.1, \phi=25^{\circ}, z_{w}^{*}=0.1$ and $k_{h}=0.1$. From the Fig.5, it can be observed that $F_{s}$ decreases with the increase of $q^{*}$ for all three cases, it is greater than unity for any value of $k_{v}$ at lower values of $q^{*}$, but it decreases being higher for lower values of $q^{*}$. For example, for $k_{v}=0.1, F_{s}$ decreases by 0.25 for an increase in $q^{*}$ from 0 
to 0.5 , whereas decrease in $F_{s}$ is 0.1 as $q^{*}$ increases from 0.5 to 1 . It is also noted that $k_{v}$ can be helpful to improve the value of $F_{s}$, and $F_{s}$ increases less with the increase of $k_{v}$ for any $q^{*}$. Taking into account of $k_{h}$ and $k_{v}, F_{s}$ can increase 0.2 than only considered $k_{h}$ when compared Fig.5 and Fig.4.

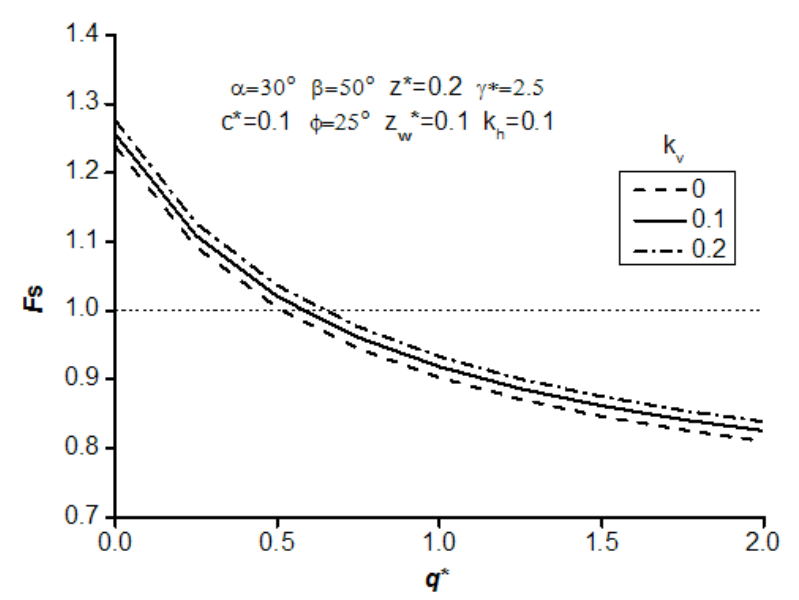

Fig. 5. Variation of $F_{s}$ with $q^{*}$ for different values of $k_{v}$

From Fig.2 to Fig.5, it is observed that $F_{s}$ decreases with the increase of $q^{*}$ for any influence parameter and it decreases being higher for lower values of $q^{*}$. The horizontal seismic coefficient $k_{h}$ is the most important parameter effect on $F_{s}$ in the above four influence parameters. It is also noted that $F_{s}$ both decreases with the increase of $z_{w}^{*}$ and $k_{h}$, however, $F_{s}$ increases less with the increase of $k_{v}$. The change of $F_{s}$ is relatively complex when increasing the value of $z^{*}$.

\section{Conclusions}

The present study provides a general analytical expression for the factor of safety of a rock slope against plane failure, incorporating most of the practically occurring under complex conditions such as surcharge, water pressure and seismic loading. Several special cases of this expression are established, which can be found similarly to those reported in the literature.

The parametric analysis has shown that $F_{s}$ of the rock slope decreases with the increase of $q^{*}$ for the range of those parameters such as $z^{*}, z_{w}^{*}, k_{h}$ and $k_{v}$ in this paper. And $F_{s}$ decreases being higher for lower values of $q^{*}$ in those four cases.

The parametric analysis has also shown that the horizontal seismic coefficient $k_{h}$ is the most important parameter effect on $F_{s}$ in the above four influence parameters. It is also noted that $F_{s}$ both decreases with the increase of $z_{w}^{*}$ and $k_{h}$, however, $F_{s}$ increases less with the increase of $k_{v}$. The change of $F_{s}$ is relatively complex when increasing the value of $z^{*}$.

The general analytical expression proposed in this paper and the results of the parametric analysis can be used to carry out a quantitative assessment of the stability of the rock slopes by engineers and researchers.

\section{References}

1. Hongshuai Liu, Jingshan Bo, Dedong Liu, "Review on study of seismic stability analysis of rock-soil slopes", Earthquake Engineering and Engineering Vibration, 25 (1), 2005, pp. 164-171. (in Chinese)

2. Christopher L. Meehan, Farshid Vahedifard, "Evaluation of simplified methods for predicting earthquake-induced slope displacements in earth dams and embankments", Engineering Geology, 152, 2013, pp. 180-193.

3. Chong $\mathrm{Xu}$, Fuchu Dai, Xiwei Xu, "Wenchuan earthquake-induced landslides: an overview", Geological Review, 56 (6), 2010, pp. 860-874. (in Chinese)

4. Lirer S., "Landslide stabilizing piles Experimental evidences and numerical interpretation", Engineering Geology, 149-150, 2012, pp. 70-77.

5. Janusz Konkol, "Selected aspects of the stability assessment of slopes with the assumption of cylindrical slip surfaces", Computers and Geotechnics, 37, 2010, pp. 796-801.

6. Yueping Yin, Yongshuang Zhang, Yinsheng Ma, et al, "Research on major characteristics of geohazards induced by the Yu Shu Ms 7.1 earthquake", Journal of Engineering Geology, 18 (3), 2010, pp. 289-296. (in Chinese)

7. Hongshuai Liu, Jingshan Bo, Dedong Liu, "Development on study of seismic stability evaluation methods of rock-soil slopes", Journal of Institute of Disaster-Prevention Science and Technology, 9 (3), 2007, pp. 20-27. (in Chinese).
8. Sharma S., Raghuvanshi T.K., Anbalagan R., "Plane failure analysis of rock slopes", Geotechnical and Geological Engineering, 13, 1995, pp. 105-111.

9. Gholamreza Mesri, Nejan Huvaj-Sarihan, "Residual shear strength measured by laboratory tests and mobilized in landslides", Journal of Geotechnical and Geoenvironmental Engineering, 138, 2012, pp. 585-593.

10. Caltabiano S., Cascone E., Maugeri M., "Static and seismic limit equilibrium analysis of sliding retaining walls under different surcharge conditions", Soil Dynamics and Earthquake Engineering, 37,2012 , pp. $38-55$.

11. Goodman R. E., Kieffer D. S., "Behaviour of rocks in slopes", Journal of Geotechnical and Geoenvironmental Engineering, 126 (8), 2000, pp. 675-684.

12. Shukla S. K., Hossain M. M., "Stability analysis of multidirectional anchored rock slope subjected to surcharge and seismic loads", Soil Dynamics and Earthquake Engineering, 31, 2011, PP. 841-844.

13. Ling H. I., Cheng A. H. D., "Rock sliding induced by seismic force", International Journal of Rock Mechanics and Mining Sciences, 34 (6), 1997, pp. 1021-1029.

14. Qiang Luo, Liang Li, Lianheng Zhao, "Quasi-static analysis of seismic stability of anchored rock slope under surcharge and water pressure conditions", Rock and Soil Mechanics, 31 (11), 2010, pp. 3585-3593. (in Chinese) 